\title{
An on-line applet-based optics course for undergraduate students
}

\author{
A. Carnicer, S. Bosch, J. Ferré, I. Juvells, I. Labastida, E. Martín-Badosa, J. R. de F. Moneo, \\ E. Pleguezuelos, R. Tudela, and S. Vallmitjana \\ Grup d'Innovació Docent en Òptica Física i Fotònica \\ Universitat de Barcelona. Departament de Física Aplicada i Òptica \\ Avda. Diagonal 647, E08028 Barcelona. E-mail: artur@fao.ub.es
}

\begin{abstract}
We present an educational resource based in a virtual optical laboratory for undergraduate students. It consists in a webbased textbook with several applets to illustrate the theory and simplify the teaching tasks in the classroom. These programs can also be used as a method for self-learning in an on-line environment. Applets are written in Java language using the Java Network Launching Protocol (JNLP) for avoiding problems related with the use of specific browsers or Java interpreters versions.
\end{abstract}

Keywords: education in optics, on-line training.

\section{THE JAVA OPTICS COURSE PROJECT}

We are currently developing an optics textbook with ordinary resources, i.e. text, equations and images that can be accessed by means of the network. The use of such technology allows the inclusion of simulations in the textbook, so the student can try, play or analyze what happen with physical phenomena when the parameters are changed. The main difficulty of our students in their learning is the understanding of theoretical concepts related with experimental situations. As a consequence, the goal of this project is to generate a small number of sophisticated programs that allows a student to interact with all the significant concepts studied in an undergraduate optics course.

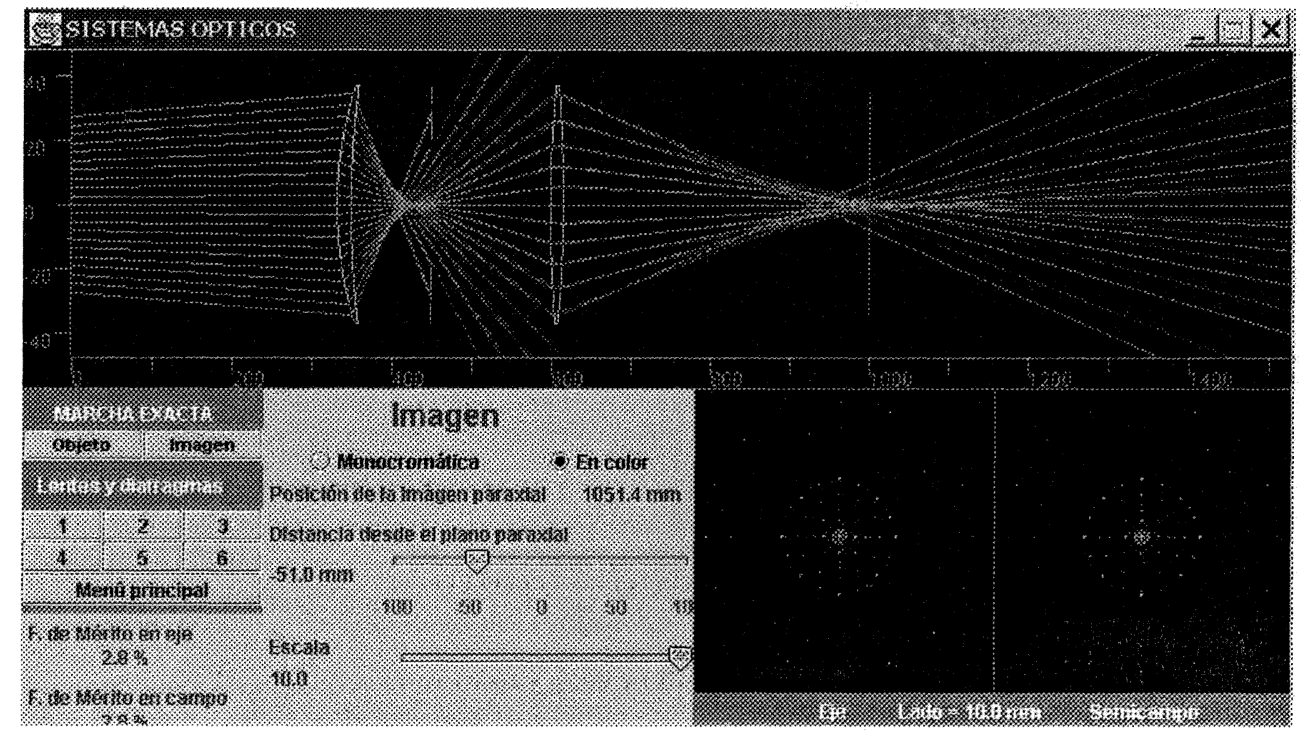

Figure 1: Optical systems design applet

The applets that are being developed are: ray tracing and optical instruments design, eye and human vision models, electromagnetic waves polarization, refraction and reflection of light, and Fresnel formulas, wave propagation in crystals, the Young experiment, Michelson and Mach-Zehnder interferometry, interferences in thin dielectric films, multilayer filter design, Fresnel and Fraunhofer diffraction, beam propagation, colorimetry, etc. The applets are going to be programmed in Java using JNLP' technology to assure a correct behavior of the programs with independence of the software or operating system installed in the computer. 
As an example of the carried out work, we present several frames of the programs. Figures 1 and 2 show screen captures of two applets developed by our group. The first one is a simulation program for designing optical systems. Ray tracing is performed in realistic conditions, avoiding paraxial approximation or taking into account the influence of wavelength over the refractive index, for instance.

In Figure 2 we show a program to simulate the intensity of a wave front when the light is diffracted in Fresnel or Fraunhofer conditions. The student can interact with the program, changing all the parameters required to perform the calculations. The diffraction image can be processed: for instance, contrast can be modified and intensity profile can be displayed for each row or column.

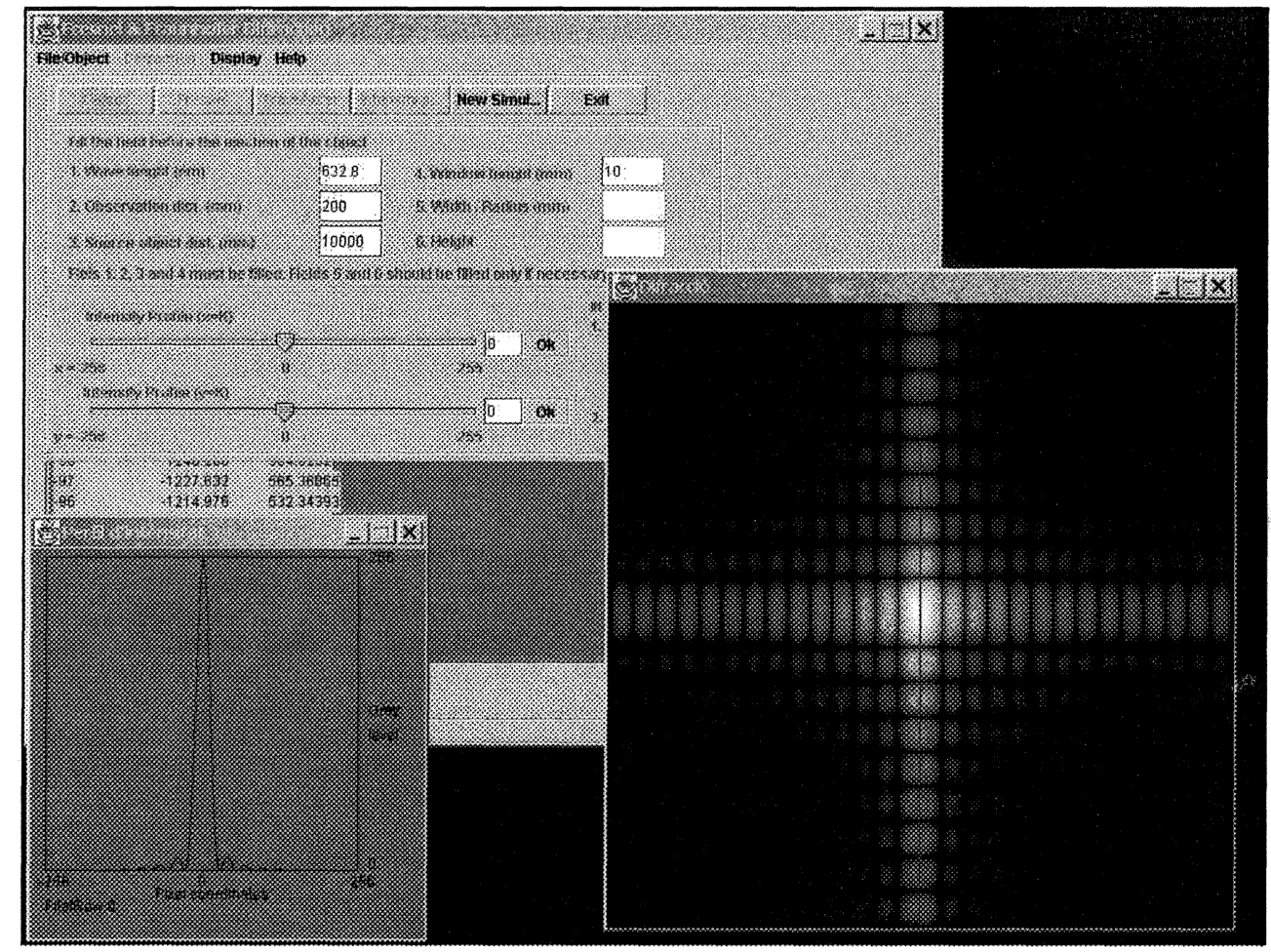

Figure 2: Fresnel and Fraunhofer diffraction applet

The students can manipulate the simulations following the indications of a list of activities. These exercises are also included in the package.

\section{ACKNOWLEDGEMENTS}

This project is funded by the Direcció General d'Universitats of the Generalitat de Catalunya and the Gabinet d'Avaluació i Innovació Universitària of the Universitat de Barcelona.

\section{REFERENCES}

1. See, for instance, http://java.sun.com/products/javawebstart/index.html 